\title{
FAM46B Promotes Apoptosis and Inhibits Glycolysis of Prostate Cancer Through Inhibition of the MYC-LDHA Axis
}

This article was published in the following Dove Press journal: OncoTargets and Therapy

\section{Tao Liang* \\ Xuxiao Ye* \\ Dongliang Yan \\ Chao Deng \\ Zuowei Li \\ Binqiang Tian}

Department of Urology, Shanghai Sixth People's Hospital East Affiliated to Shanghai University of Medicine \& Health Sciences, Shanghai 20I306, People's Republic of China

*These authors contributed equally to this work
Correspondence: Dongliang Yan Email Liang_six@163.com
Objective: Increased dependence on glycolysis is a known element of cancer. This study was designed to examine critical glycolysis components including transcription factor MYC and its downstream target lactate dehydrogenase A (LDHA), potential upstream regulators of glycolysis such as family with sequence similarity 46 member B (FAM46B), and the impact of the abundance of these proteins on apoptosis and glycolysis in prostate cancer.

Materials and Methods: A total of 70 primary prostate cancer patient samples were compared to normal tissues for FAM46B and LDHA expression and the corresponding patients' survival was monitored for 60 months. Prostate cancer cell lines were employed for protein expression manipulation, glucose uptake and LDH assays, and apoptosis measurements. A xenograft mouse model was used to quantify the role of FAM46B and LDHA on tumor growth in vivo.

Results: FAM46B expression was reduced in prostate tumor tissue compared to normal tissue and prostate cancer patients who expressed low amounts of FAM46B had shortened average lifespans compared to those who expressed higher amounts of FAM46B ( $p=0.008)$. FAM46B overexpression reduced glucose uptake, decreased LDH activity, and induced apoptosis in prostate cancer cell lines while FAM46B shRNA increased MYC levels in a non-malignant prostate cell line (P69). Conversely, forced expression of LDHA in LNCaP cells produced an increase in glycolysis markers with a corresponding decrease in apoptosis. FAM46B-overexpressing xenografts had starkly blunted growth which was restored with cooverexpression of LDHA.

Conclusion: FAM46B plays a central role in regulating glycolysis and apoptosis in prostate cancer and operates through the regulation of LDHA via MYC. FAM46B's keystone status in prostate cancer makes it a potential, robust biomarker for prostate cancer prognosis and a promising therapeutic target.

Keywords: prostate cancer, LDHA, FAM46B, glycolysis

\section{Introduction}

Prostate cancer is a common disease, particularly for older men. In 2018, 1,276,106 incidences of prostate cancer diagnoses were made worldwide with the majority of men over the age of 60 developing the disease. ${ }^{1}$ The rate of diagnosis is a particular concern in countries with large aging populations. ${ }^{2}$ Importantly, prostate cancer incidences are increasing, highlighting the need for both improved sensitivity in detection and more efficacious treatments. The risk of death from prostate cancer is exacerbated by its asymptomatic nature in which symptoms present only in the late 
stages of cancer when metastasis occurs. ${ }^{3}$ Due to the very high prevalence of prostate cancer, its asymptomatic nature, and fatality risk, avenues for both improved treatment and detection need to be developed.

An attractive target for both the diagnosis and treatment of prostate cancer is Family with sequence similarity 46 member B (FAM46B). FAM46B is a protein belonging to a family of four related proteins (FAM46A, FAM46B, FAM46C, and FAM46D) which have largely undefined functions but have been shown to play a role in tumorigenesis. Specifically, a Single Nucleotide Polymorphism (SNP) found in FAM46A has been correlated with breast cancer, ${ }^{4}$ a tandem repeat in the FAM46A gene has been found in non-small cell lung cancer ${ }^{5}$ and FAM46A expression is decreased in prostate cancer. ${ }^{6}$ FAM46B has been shown to reduce prostate tumor growth in an in vivo model through ubiquitination of $\beta$-catenin. ${ }^{7}$ FAM46C has been found to have tumor-suppressing functions in multiple myeloma ${ }^{8,9}$ as well as hepatocellular carcinoma. ${ }^{10-12}$ Finally, FAM46D is a proposed cancer immunotherapy target. $^{12}$

Tumor cells typically survive in a hypoxic, highly metabolic state. Because of this, and other possible unknown mechanisms, cancer cells favor glycolysis over oxidative phosphorylation to generate energy (the Warburg effect). ${ }^{13}$ It is speculated that the expression of lactate dehydrogenase A (LDHA) is increased in cancer as part of a system to fuel the cellular metabolism of these cells. Indeed, LDHA is dysregulated in several cancers including pancreatic cancer, ${ }^{14,15}$ breast cancer, ${ }^{16,17}$ and glioma, ${ }^{18}$ plays a role in the aggressiveness of gastric cancer, ${ }^{19}$ and controls apoptosis in some cancer types. ${ }^{20}$ Expression of LDHA is speculated to be regulated by a few known factors, including fibroblast growth factor receptor, ${ }^{21}$ cyclin G2, ${ }^{22}$ FLF4, ${ }^{23}$ FOXM1, ${ }^{24}$ or through a welldefined MYC-LDHA pathway; ${ }^{14,25,26}$ however, there are little data beyond these, particularly in regards to upstream initiators.

The function of the MYC/LDHA pathway is critical for the maintenance of the Warburg effect. ${ }^{27}$ Specifically, it is believed that MYC-mediated LDHA expression is a primary component in lactate/pyruvate cycling in tumor cells. It is through these LDHA-dependent pathways that tumors are able to survive and proliferate.

In this study, we examine the role of FAM46B in the regulation of apoptosis and glycolysis in prostate cancer and the possible molecular mechanism involved in the MYC-LDHA axis. We show that FAM46B is downregulated in primary patient prostate cancer cells and that low FAM46B expression correlates with poor patient survival. We also show that intentional deregulation of FAM46B reduces glycolysis and induces apoptosis in prostate cancer cells while FAM46B shRNA increases glycolysis markers in a non-tumorigenic prostate cell line. Finally, we demonstrate that LDHA is a regulatory target of FAM46B in prostate cancer cells and suggest a role for MYC in mediating this regulation.

\section{Materials and Methods}

\section{Clinical Samples}

Thirty-five cases of tumor tissue and 35 cases of neighboring noncancerous tissue were received from prostate cancer patients in Shanghai Sixth People's Hospital East Affiliated to Shanghai University of Medicine \& Health Sciences recruited from March 2014 to October 2017. The human prostate cancer tissue microarrays used in this study were prepared by Shanghai Outdo Biotech Co., Ltd. (Shanghai, China) and collected for the measurement of FAM46B protein abundance by immunohistochemistry (IHC) analysis, and detailed information is presented in Table 1. All of the patients provided signed informed

Table I Association of FAM46B Expression with Clinical Characteristics of 70 Patients with Prostate Cancer

\begin{tabular}{|c|c|c|c|c|}
\hline \multirow[t]{2}{*}{ Characteristics } & \multirow{2}{*}{$\begin{array}{l}\text { Patients } \\
(n=70)\end{array}$} & \multicolumn{2}{|l|}{ FAM46B } & \multirow[t]{2}{*}{$P$} \\
\hline & & $\begin{array}{l}\text { High } \\
(n=4 I)\end{array}$ & $\begin{array}{l}\text { Low } \\
(n=29)\end{array}$ & \\
\hline Age & & & & 0.234 \\
\hline$<70$ & 40 & 21 & 19 & \\
\hline$>70$ & 30 & 20 & 10 & \\
\hline Tumor size $(\mathrm{cm})$ & & & & 0.018 \\
\hline$<3$ & 31 & 23 & 8 & \\
\hline$>3$ & 39 & 18 & 21 & \\
\hline Tumor stage & & & & 0.555 \\
\hline pT2 & 53 & 30 & 23 & \\
\hline pT3/pT4 & 17 & 11 & 6 & \\
\hline TNM stage & & & & 0.005 \\
\hline 1 & 20 & $16(39.0)$ & 4 & \\
\hline II & 34 & $14(34.1)$ & 20 & \\
\hline III & 12 & $10(24.4)$ & 2 & \\
\hline IV & 4 & I (2.5) & 3 & \\
\hline Gleason score & & & & 0.132 \\
\hline$\leq 6$ or $=3+4$ & 24 & $17(4 \mid .5)$ & 7 & \\
\hline$=4+3$ or $\geq 8$ & 46 & $24(58.5)$ & 22 & \\
\hline
\end{tabular}

Note: Differences between groups were done by the Chi-square test. 
consent. The medical ethics committee of Shanghai Sixth People's Hospital East Affiliated to Shanghai University of Medicine \& Health Sciences approved the present retrieval method of cancer specimens.

\section{Immunohistochemistry (IHC)}

Paraffin-embedded tissue sections of primary prostate cancer and the bordering non-tumor prostate tissues were employed for immunohistochemical analysis. IHC staining of FAM46B protein was implemented on prostate cancer specimens following a conventional protocol using an antiFAM46B antibody (Biorbyt), followed by incubation with a secondary antibody (Shanghai Long Island Biotec. Co., Ltd). Immunohistochemical evaluation was performed by two pathologists blinded to the clinical characteristics of the patients. Based on IHC, patients with $25 \%$ or more of tumor cells with positive staining were categorized as the FAM46B high expression group and those with fewer than $25 \%$ of tumor cells with positive staining were placed into the FAM46B low expression group.

\section{Cell Culture and Transfection}

PC3 and LNCaP human prostate cancer cell lines and P69 prostate epithelial cell lines were purchased from the cell bank of Shanghai Biology Institute, Chinese Academy of Science (Shanghai, China). LNCaP, PC3 and P69 cells were cultured in Dulbecco's modified Eagle's medium (DMEM). All culture media was supplemented with 10\% fetal bovine serum and 1\% penicillin/streptomycin (Life Technologies). Cells were culture in $5 \% \mathrm{CO}_{2}$ at $37^{\circ} \mathrm{C}$.

To elevate the expression of FAM46B and LDHA, the coding sequence was synthesized and cloned into pLVXPuro plasmids (Clontech, USA). RNA interference sequences targeting FAM46B were manufactured and cloned into linearized pLKO.1 plasmids (Addgene, USA). The interference sites and corresponding primer sequences are as follows: FAM46B (shRNA-1, position 358-376 5'-TCTTCTGAGCGAGCCGATT-3'; shRNA-2, position 762-780, 5'-GCAAGAACGTGGAGCTCAA-3'). Recombinant lentiviral plasmids with packaging plasmids psPAX2 and pMD2G were co-transfected into 293T cells using Lipofectamine 2000 (Invitrogen, USA). At $48 \mathrm{~h}$ post transfection, virus-containing supernatants were collected and used for transducing cells. Cells with pLKO.1-scramble shRNA or empty pLVX-Puro vectors were used as negative controls.

\section{Cell Apoptosis Assay}

Cells were seeded into a six-well plate $\left(5 \times 10^{5} /\right.$ well $)$ and grown to $50 \%$ confluence. Collected LNCaP or PC3 cells were transduced with pLVX-Puro-FAM46B and/or pLVXPuro-LDHA vector, and P69 cells were transduced with pLKO.1-shRNA-FAM46B vector. At $48 \mathrm{~h}$ post-transduction, apoptosis was measured using flow cytometry. Briefly, cells were labeled with $5 \mu \mathrm{L}$ fluorescein isothiocyanate-labeled recombinant annexin $\mathrm{V}$ (Annexin V-FITC) for $15 \mathrm{~min}$ in the dark at $4^{\circ} \mathrm{C}$, followed by $5 \mu \mathrm{L}$ PI for another $15 \mathrm{~min}$.

\section{Measurement of Glucose Uptake}

Glucose uptake was measured using a fluorescent glucose 2-NBDG (2-Deoxy-2-[(7-nitro-2,1,3)]-benzoxadiazol-4-yl) Glucose Uptake Assay Kit (Biovision, Milpitas, CA, USA) following the manufacturer's protocol. In brief, $5 \times 10^{5}$ cells per well in a six-well plate were cultured at $37^{\circ} \mathrm{C}$ for $24 \mathrm{hr}$. Collected LNCaP or PC3 cells were transduced using pLVX-Puro-FAM46B and/or pLVX-Puro-LDHA vector, and P69 cells were transduced with pLKO.1-shRNAFAM46B vector. At $48 \mathrm{~h}$ after transduction, cells were starved for glucose for $3 \mathrm{~h}$. After incubating with KrebsRinger Bicarbonate Buffer with 2\% bovine serum albumin (BSA) for $40 \mathrm{~min}, 2-\mathrm{NBDG}(100 \mu \mathrm{M})$ was injected into each well and incubated for $45 \mathrm{~min}$ at $37^{\circ} \mathrm{C}$. The cells were then washed with PBS, trypsinized, resuspended in $10 \%$ FBS before subjecting to flow cytometry analysis. The glucose uptake potential of the samples was analyzed using BD Accuri C6 flow cytometer (BD Biosciences, Franklin Lakes, NJ, USA).

\section{Measurement of Lactate}

$5 \times 10^{5}$ cells per well in a six-well plate were cultured for $24 \mathrm{hrs}$ at $37^{\circ} \mathrm{C}$. Collected LNCaP or PC3 cells were transduced with pLVX-Puro-FAM46B and/or pLVX-PuroLDHA vector, and P69 cells were transduced with pLKO.1-shRNA-FAM46B vector. At $48 \mathrm{~h}$ after transduction, cellular lactate release was measured using the Lactic Acid assay kit (Nanjing Jiancheng Bioengineering Institute, China) according to the kit manual.

\section{Quantitative RT-PCR}

TRIzol reagent (Life Technologies, Inc., Waltham, MA, USA) was used to extract RNA from prostate cancer tissues and cell lines. cDNA was produced from RNA via PrimeScript kit (Takara Biotechnology, Dalian, China) following the manufacturer's protocol. SYBR green quantitative RT-PCR was 
performed in an ABI 9700 real-time PCR system (Applied Biosystem) using standard reagents (Applied Biosystems, Foster, CA, USA). The primers used for the PCR are as follows: FAM46B-F: 5'-CTGGCTGCCTTGTAACTG-3', FAM46B-R: 5'-TCGGGAAAGTCTGGTCTG-3'; LDHA-F: 5'-AGCCCGATTCCGTTACCTAATG-3', LDHA-R: 5'-ACC TGCTTGTGAACCTCTTTCC-3'; GAPDH-F: 5'-AATCCC ATCACCATCTTC-3', GAPDH-R: 5'-AGGCTGTTGTC ATACTTC-3'. Fold change of FAM46B and LDHA mRNA was determined by the $2^{-\Delta \triangle \mathrm{CT}}$ method.

\section{Western Blot Analysis}

Protein was extracted in RIPA lysis buffer with protease inhibitor cocktail (Sigma, St. Louis, MO, USA), separated by SDS-PAGE, and transferred onto a nitrocellulose membrane (Millipore, Bedford, USA). Membranes were blocked with $5 \%$ reconstituted dry milk, and incubated with antibodies against FAM46B (Biorbyt; Orb354867), cleaved caspase-3 (Abcam; ab2302), LDHA (Abcam; ab101562), MYC (Abcam; ab32072) and GAPDH (Cell Signaling Technology, Danvers, MA, USA; \#5174) then incubated with an HRP-conjugated secondary antibody (Beyotime, Shanghai, China; A0208) following manufacturer's protocol. Protein bands were visualized with an enhanced chemiluminescence system (Bio-Rad, Richmond, CA, USA).

\section{Establishment of Stable Cell Lines and Xenograft Study}

LNCaP cells $\left(5 \times 10^{6}\right)$ transduced with pLVX-PuroFAM46B, pLVX-Puro-LDHA, or empty vector were subcutaneously injected into the axilla of 4- to 5-week-old male nude mice (Shanghai Laboratory Animal Company, Shanghai, China). Tumors were measured every 3 days and tumor volumes were calculated using the formula: Tumor volume $=\left(\right.$ length $\mathrm{x}$ width $\left.{ }^{\wedge}\right) / 2$. Thirty-three days after injection, mice were sacrificed and tumor xenografts were collected, photographed and weighed $(n=6$ per group). The xenografts were subjected to TUNEL (Terminal deoxynucleotidyl transferase dUTP Nick-End Labeling) analyses (Roche, Indianapolis, IN, USA). Care of the laboratory animals and animal experimentation were performed in accordance with the animal ethics guidelines and approved protocols of Shanghai Sixth People's Hospital East Affiliated to Shanghai University of Medicine \& Health Sciences (YS-2019-089).

\section{Statistical Analysis}

All experiments were carried out three times and the quantitative data were expressed as mean $\pm \mathrm{SD}$. All statistics were analyzed by GraphPad Prism 7.0 (GraphPad Software, San Diego, CA, USA). A comparison between different experimental groups was performed using ANOVA followed by Tukey's post-multiple test. $P<0.05$ was considered to have a significant statistical difference. Kaplan-Meier method and Cox's proportional hazards regression model were used to calculate overall survival and the differences were analyzed by a Log rank test.

\section{Results}

\section{FAM46B is Downregulated in Prostate Cancer Tissues and Correlated with Poor Survival}

Clinical samples were collected from hospitals following informed consent. The expression of FAM46B in prostate cancer tissues was analyzed by qRT-PCR (Figure 1A) and IHC (Figure 1B). IHC samples were quantified based on the percentage of positive staining (Figure 1B, lower) and the association of FAM46B expression according to the IHC analysis (high vs low) with clinicopathological characteristics was analyzed (Table 1). FAM46B expression was associated with tumor size and TNM stage, but not with age, tumor stage, or Gleason score. A comparison of 70 prostate cancer patients presenting high FAM46B expression $(n=41)$ with those presenting low FAM46B expression $(\mathrm{n}=29)$ showed a significant $(p=0.008)$ lower overall survival over a 60-month period for patients with low FAM46B expression (Figure 1C). A breakdown of potential prognostic (Figure 1D) factors found the highest hazard ratio (HR) between FAM46B expression and survival $(\mathrm{HR}=1.50)$, followed by Gleason Score $(\mathrm{HR}=1.28)$, TNM stage $(\mathrm{HR}=1.19)$, tumor size $(\mathrm{HR}=1.16)$, age $(\mathrm{HR}=$ $0.95)$, and tumor stage $(\mathrm{HR}=0.75)$, indicating the potential for FAM46B as a prognostic factor.

\section{Overexpression of FAM46B Promotes Apoptosis and Inhibits Glycolysis of LNCaP and PC3 Cells}

Previous studies have found that FAM46B abundance was significantly higher in the non-tumorigenic P69 cell line compared to the tumorigenic $\mathrm{LNCaP}$ and PC3 cell lines. ${ }^{3}$ We increased the FAM46B levels using an expression vector and confirmed overexpression and protein 
A

FAM 46B m RNA level $(P<0.001)$

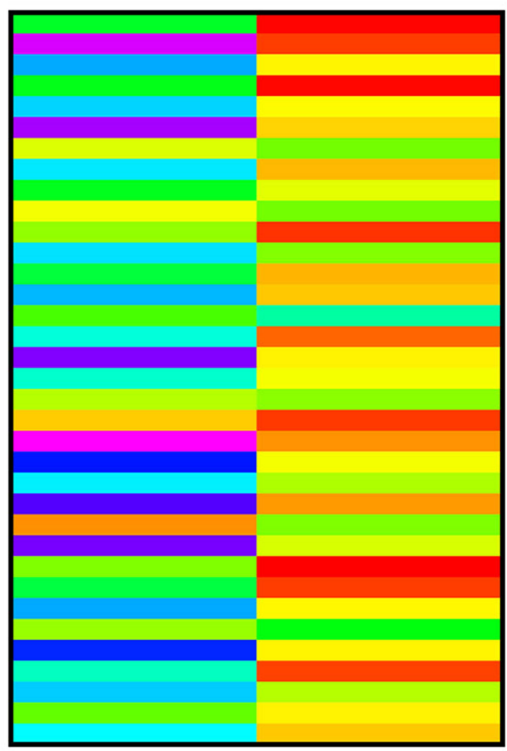

$N(n=35)$

T $(n=35)$

C

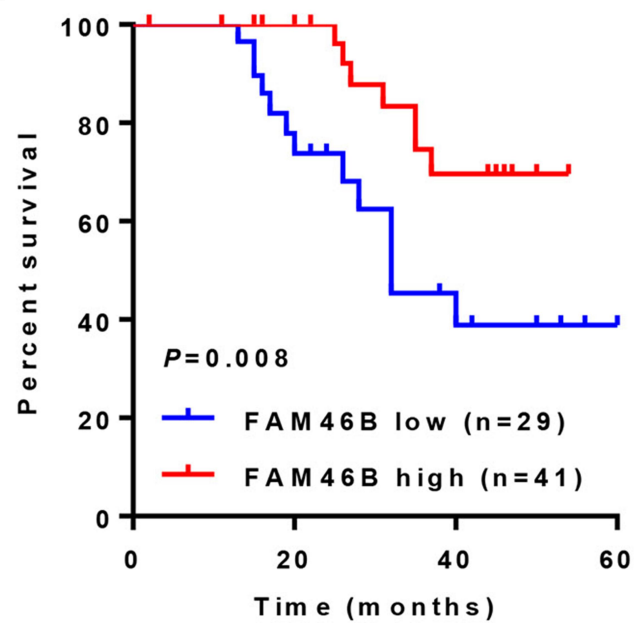

B

$N(n=10)$

$T(n=70)$
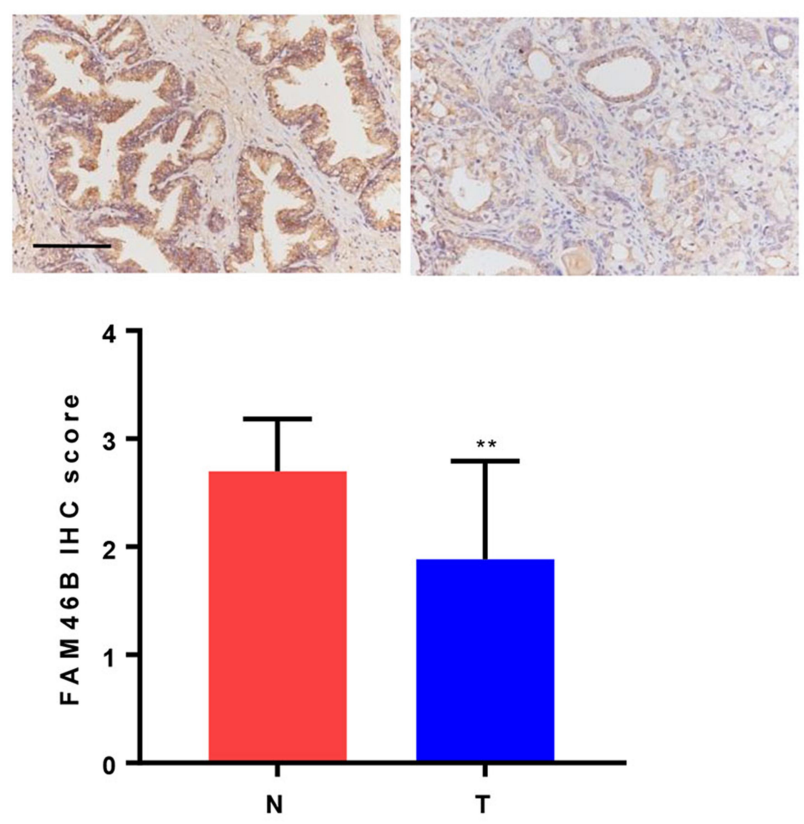

D

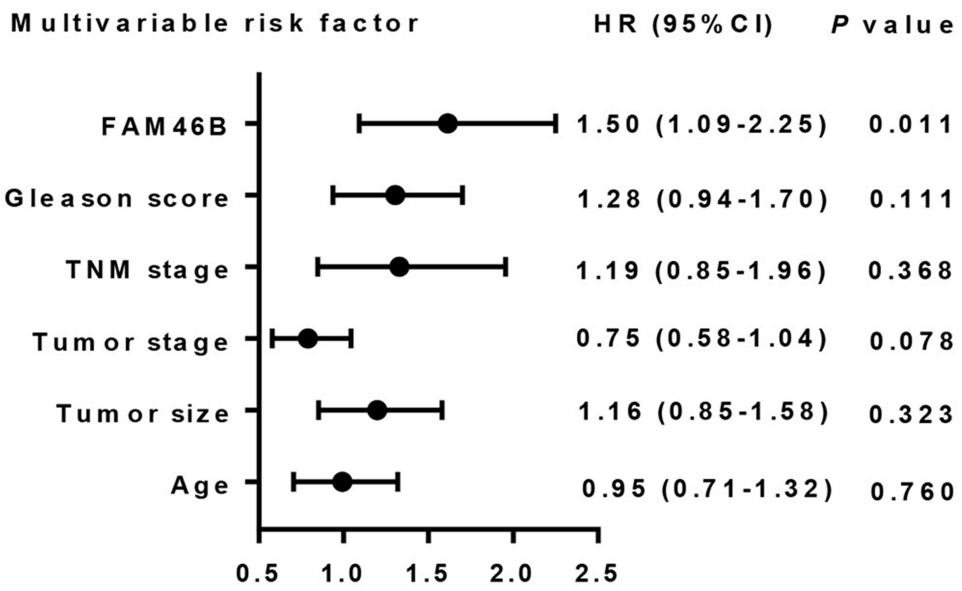

Figure I FAM46B expression in prostate cancer tissues. (A) FAM46B mRNA expression in 35 prostate cancer (T) and paired normal (N) tissues from the hospital cohort. (B) Typical images of immunohistochemistry $(\mathrm{IHC})$ in 70 prostate cancer and 10 normal tissues from tissue microarrays showing the protein expression of FAM46B. Scale bar: $100 \mu \mathrm{m}$. (C) Kaplan-Meier curves for overall survival rates of prostate cancer patients according to FAM46B expression levels based on IHC analysis. (D) Multivariate analysis of overall survival in prostate cancer patients. $* * P<0.01$ compared with $\mathrm{N}$.

abundance by quantitative RT-PCR and by Western blot (Figure 2A). Annexin V/PI staining indicated that overexpression of FAM46B induced apoptosis in $\mathrm{LNCaP}$ and PC3 cells, respectively, compared to empty vector group (Figure 2B), confirming the suspected role of FAM46B in apoptosis. Glucose uptake, gauged by 2-NBDG uptake, was determined to be decreased in LNCaP and PC3 cells overexpressing FAM46B (Figure 2C). Accordingly, both lactate and $\mathrm{LDH}$ activity were found to be reduced in both LNCaP and PC3 cells following FAM46B overexpression
(Figure 2D, and E). To demonstrate the role of FAM46B in the regulation of apoptosis and cell survival, we measured the protein abundance of the pro-apoptotic protein caspase-3, the proto-oncoprotein MYC, and LDHA. In agreement with our annexin V/PI staining, both LNCaP and PC3 cells demonstrated a marked increase in cleaved caspase-3 levels, while anti-apoptotic MYC was found in lower abundance following FAM46B overexpression. LDHA levels were also reduced in FAM46Boverexpressing LNCaP and PC3 cells, in accordance 

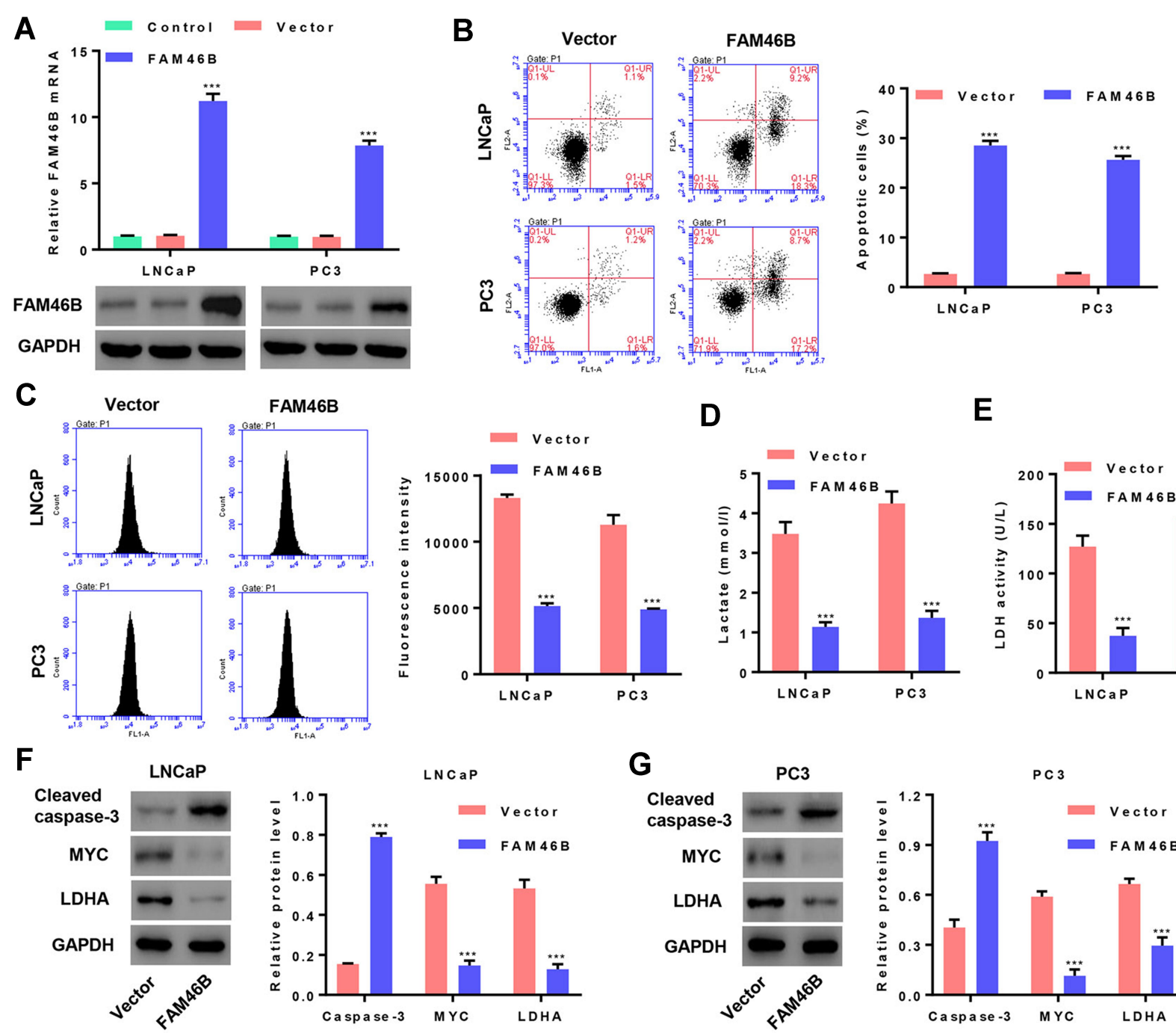

E
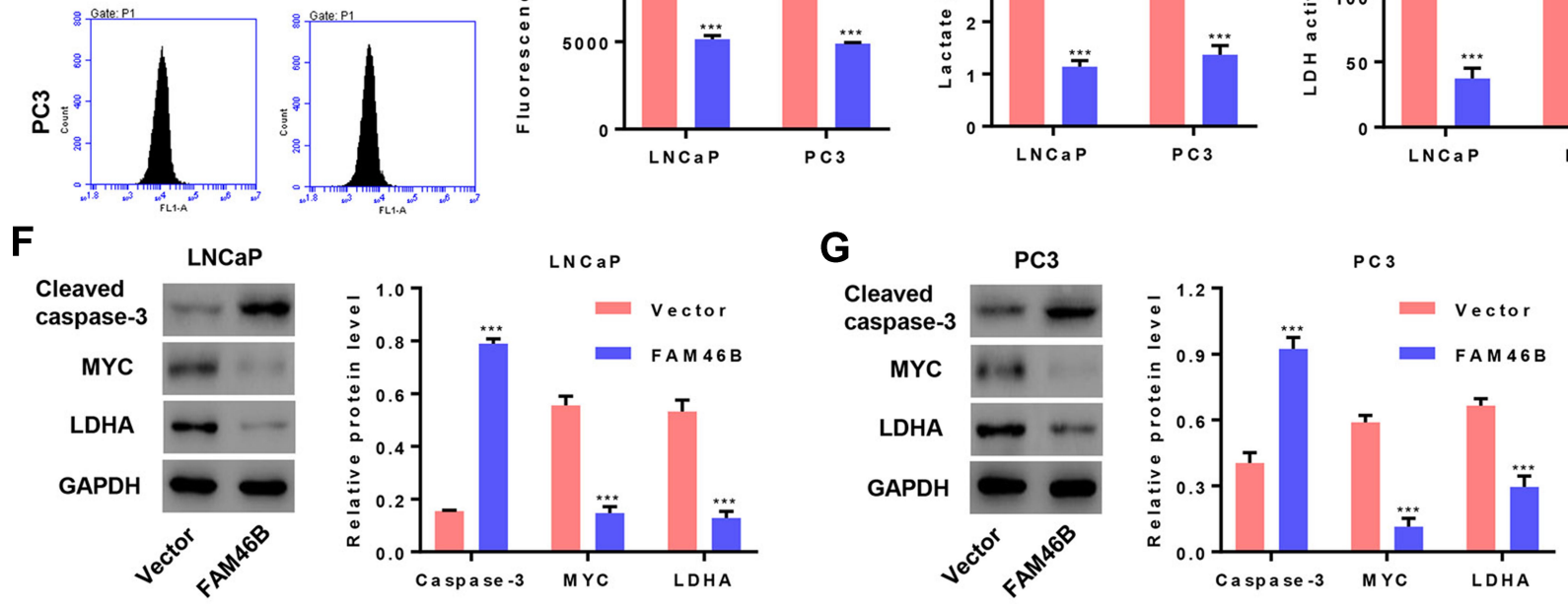

Figure 2 FAM46B overexpression promotes apoptosis and inhibits glycolysis of LNCaP and PC3 cells. (A) mRNA and protein expression of FAM46B in LNCaP and PC3 cells transduced with FAM46B expressing vector (FAM46B) or empty vector (vector). After transduction, (B and C) flow cytometry was performed to assess cell apoptosis and glucose uptake, (D) lactate release and (E) LDH activity were measured by biochemical analysis, and (F and $\mathbf{G})$ protein expression of cleaved caspase-3, MYC and LDHA was measured by Western blot. $* * * P<0.001$ compared with vector group.

with our data showing a reduction in $\mathrm{LDH}$ activity (Figure 2F and G).

\section{Knockdown of FAM46B Promotes}

\section{Apoptosis and Inhibits Glycolysis of P69 Cells}

Knockdown of FAM46B using two unique shRNA sequences in P69 cells was confirmed by quantitative RTPCR and Western blot (Figure 3A). Converse to the apoptosis induction by FAM46B overexpression, we found that baseline apoptosis in P69 cells was reduced by FAM46B knock-down by FAM46B shRNA when compared to control shRNA (Figure 3B). Glucose uptake was increased in cells expressing FAM46B shRNA (Figure 3C). Lactate release and, correspondingly, $\mathrm{LDH}$ activity were found to be increased in P69 cells with reduced FAM46B abundance (Figure 3D and E). Likewise, knock-down of FAM46B reduced cleaved caspase-3 levels while increasing both MYC and LDHA (Figure 3F).

\section{FAM46B Regulates Apoptosis and Glycolysis of LNCaP Cells Through Inhibition of LDHA}

To evaluate the FAM46B/LDHA relationship from the function of LDHA we overexpressed LDHA in LNCaP cells (Figure 4A). A comparison of apoptosis among LDHA-overexpressing LNCaP cells, LNCaP cells overexpressing FAM46B, LNCaP cells overexpressing both 
A
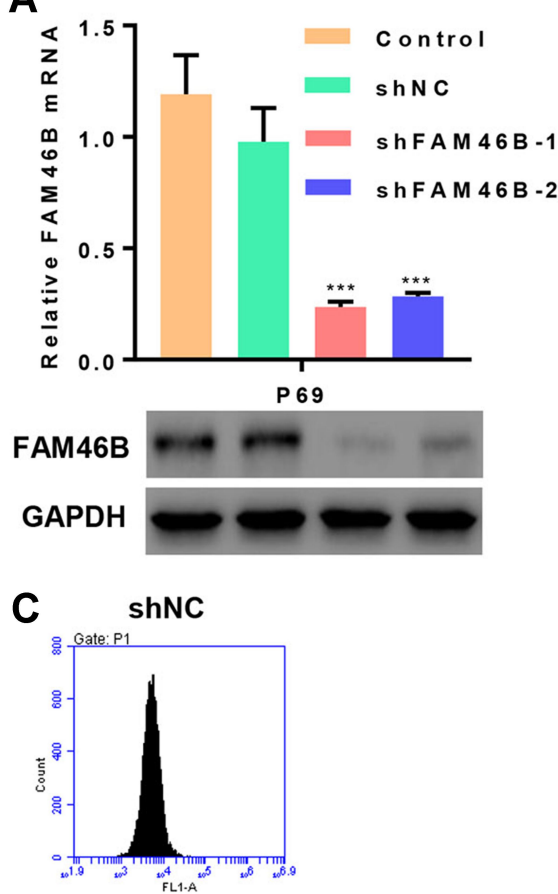

shFAM46B-1
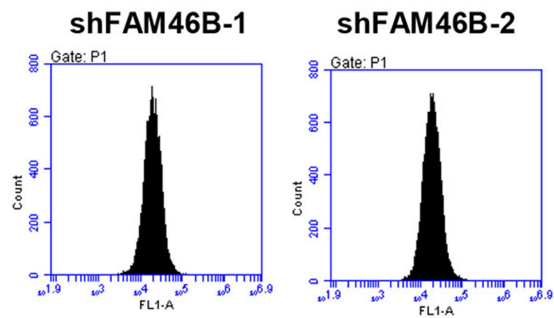

E

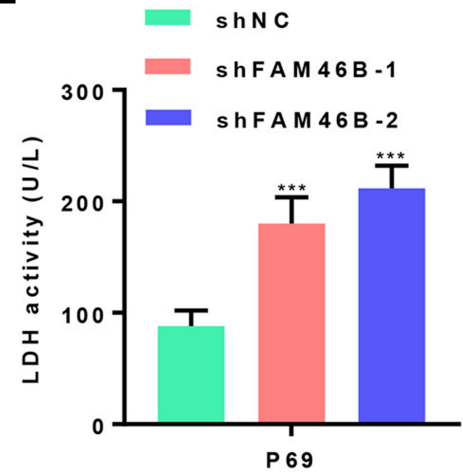

$\mathrm{F}$
B

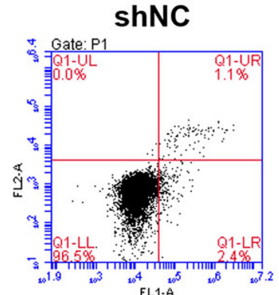

shFAM46B-1

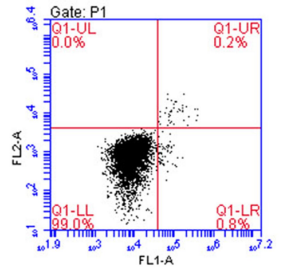

ShFAM46B-2
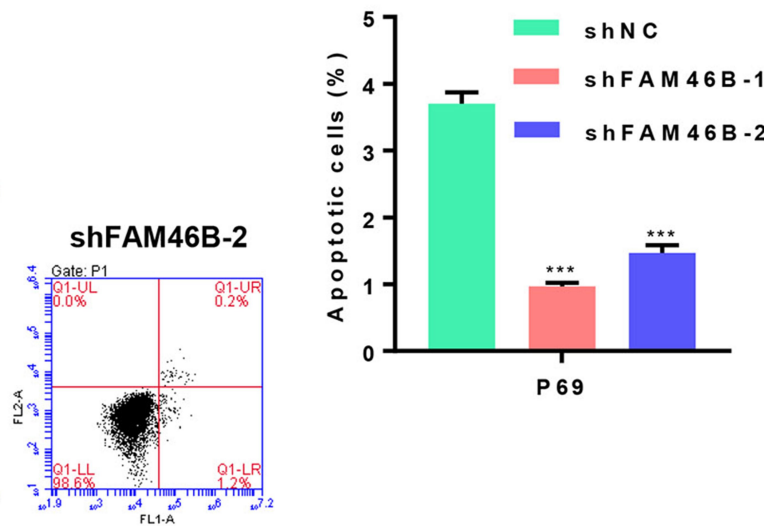
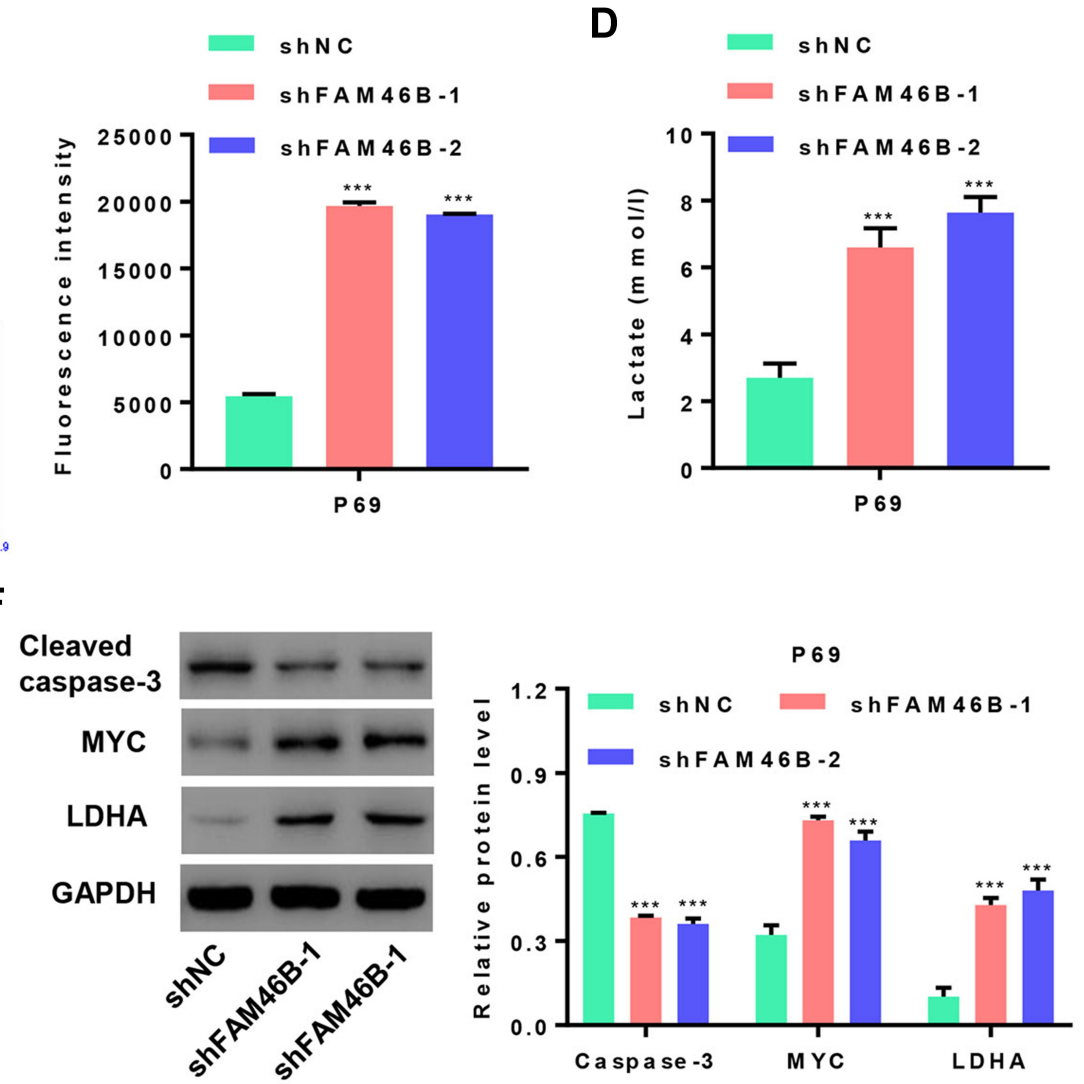

Figure 3 FAM46B silencing inhibits apoptosis and promotes glycolysis of P69 cells. (A) mRNA and protein expression of FAM46B in P69 cells transduced with FAM46B silencing vector (shFAM46B) or scramble shRNA vector (shNC). After transduction, (B and $\mathbf{C})$ flow cytometry was performed to assess cell apoptosis and glucose uptake, (D) lactate release and (E) LDH activity were measured by biochemical analysis, and (F) protein expression of cleaved caspase-3, MYC and LDHA was measured by Western blot. $* * * P<0.001$ compared with shNC group.

proteins, and empty vector cells was performed. As described previously, cells overexpressing FAM46B showed an increase in apoptosis compared to empty vector controls; LDHA-overexpressing cells showed a decrease in baseline apoptosis compared to controls. Simultaneous overexpression of both proteins resulted in a blunting of the impact of FAM46B overexpression resulting in a partial rescue of cell viability (17.4\% apoptotic cells) (Figure 4B).

Glucose uptake followed a pattern like that of apoptosis with FAM46B and LDHA performing opposing functions. While FAM46B overexpression in LNCaP cells decreased 

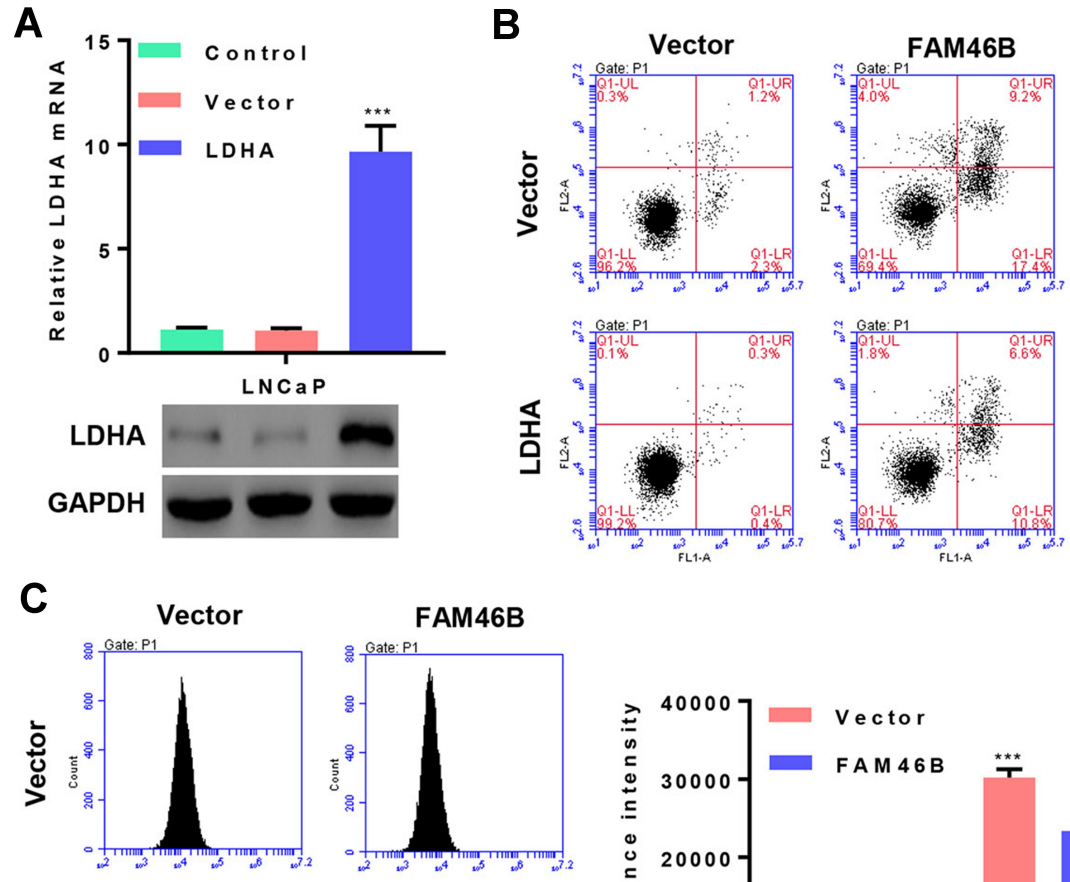

FAM46B
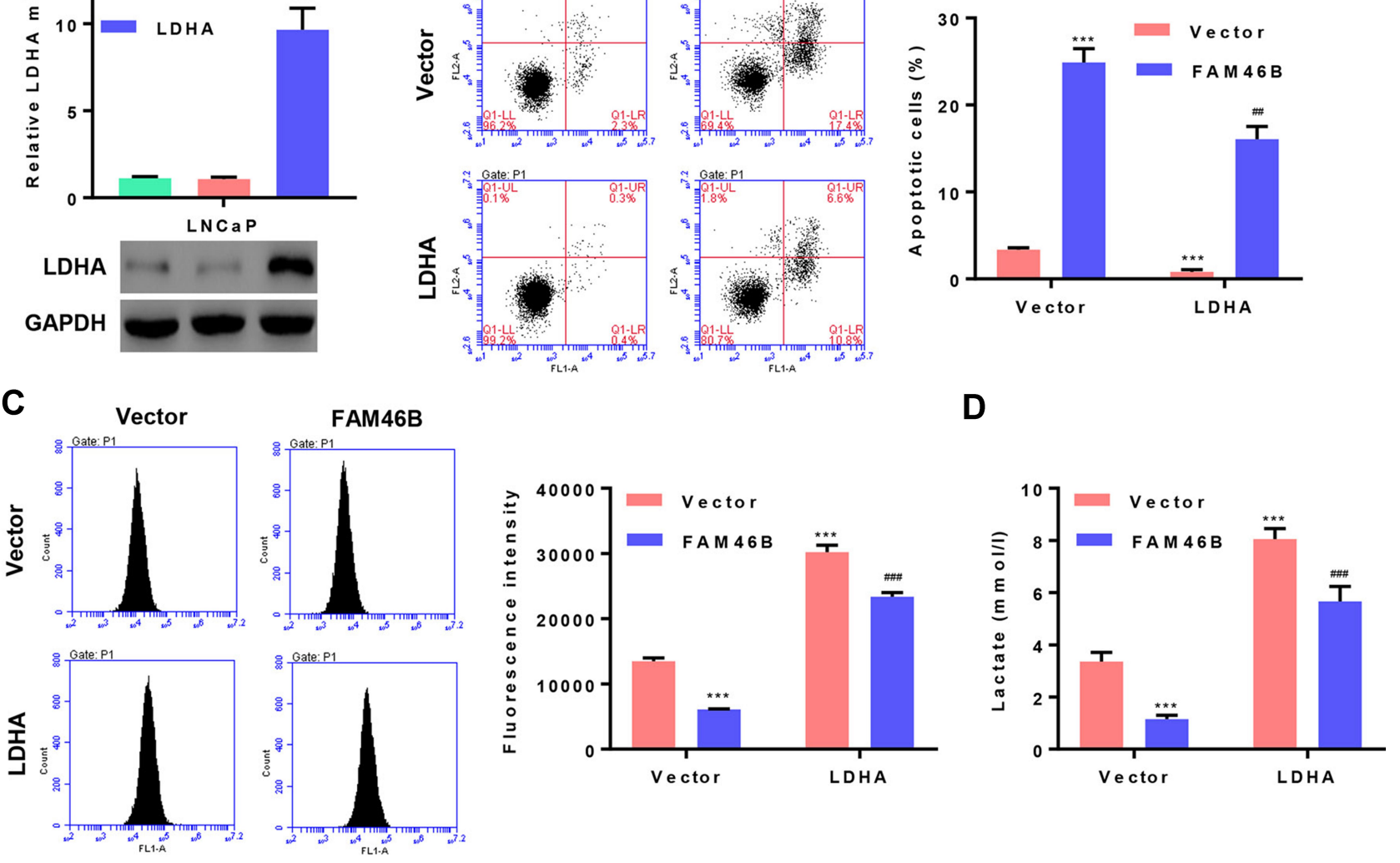

\section{E}

$\mathbf{F}$
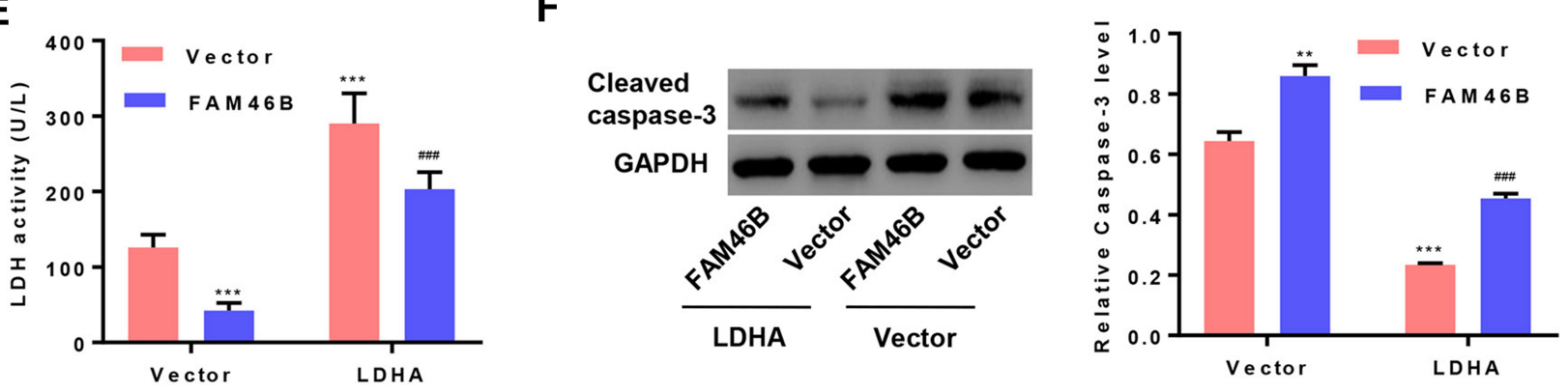

Figure 4 FAM46B promotes apoptosis and inhibits glycolysis through inhibition of LDHA in LNCaP. (A) mRNA and protein expression of LDHA in LNCaP cells transduced with LDHA expressing vector (LDHA) or empty vector (vector). After LNCaP cells were transduced with FAM46B expressing vector (FAM46B) and LDHA expressing vector (LDHA) or empty vector (vector), (B, C) flow cytometry was performed to assess cell apoptosis and glucose uptake, (D) lactate release and (E) LDH activity were measured by biochemical analysis, and (F) protein expression of cleaved caspase-3, MYC and LDHA was measured by Western blot. $* * P<0.0 \mathrm{I}$, $* * * P<0.00 \mathrm{I}$ compared with vector group. ${ }^{\#} P<0.01,{ }^{\# \#<0.001}$ compared with FAM46B group.

glucose uptake, LDHA overexpression increased glucose uptake, and co-overexpression of both proteins resulted in glucose uptake which was only modestly changed from empty vector control (Figure 4C). Both lactate release and LDH activity were expectedly increased in LDHAoverexpressing LNCaP cells; FAM46B overexpression modestly reduced both measurements. Overexpression of FAM46B in cells which also overexpress LDHA resulted in a reduction in lactate release and LDH activity when compared to cells overexpressing LDHA alone (Figure 4D and E). Cleaved caspase-3 abundance correlated with apoptosis: LDHA overexpression decreased cleaved caspase-3 abundance while FAM46B increased it. Overexpression of both proteins produced an intermediate abundance of cleaved caspase-3 (Figure 4F). Together, these data show a clear opposing mechanism between pro-apoptotic, antiglycolytic FAM46B and anti-apoptotic, pro-glycolytic LDHA. 


\section{Overexpression of FAM46B Inhibits Tumor Progression in vivo Through Inhibition of LDHA}

To validate the functions of FAM46B and LDHA in vivo, LNCaP cells with FAM46B and/or LDHA overexpression were injected into nude mice and the growth of the xenografts was observed for 33 days. FAM46B overexpression inhibited tumor growth (Figure 5A and $\mathrm{B}$ ), promoted cell apoptosis (Figure $5 \mathrm{C}$ and $\mathrm{D})$, and led to low levels of LDHA expression (Figure 5E) in xenograft tumor tissues. However, LDHA overexpression demonstrated an inverse effect. Additionally, LDHA overexpression counteracted the FAM46B overexpression-induced anti-tumor effect in vivo.

\section{Correlation Analyses in Prostate Cancer} Tissues

Primary prostate samples including tumor $(\mathrm{T})$ and local normal $(\mathrm{N})$ tissue were collected from hospital and analyzed for LDHA expression by qRT-PCR. Expression was found to be significantly higher in tumor tissues compared to normal prostate tissue $(p<0.001)$ (Figure 6A). The mRNA expression of LDHA was inversely proportional to that of FAM46B $(r=-0.4354, p=0.0089)$ (Figure 6B).

\section{Discussion}

We have examined the role of FAM46B in prostate cancer using manipulated cell lines, primary patient samples, and an in vivo xenograft model. We found FAM46B mRNA levels to be significantly lower in tumor tissues and that
A

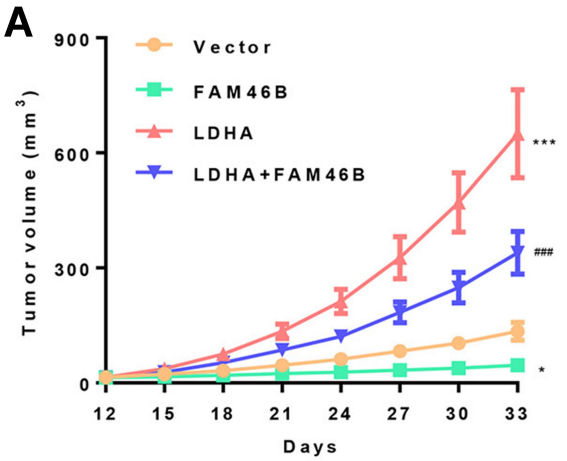

C

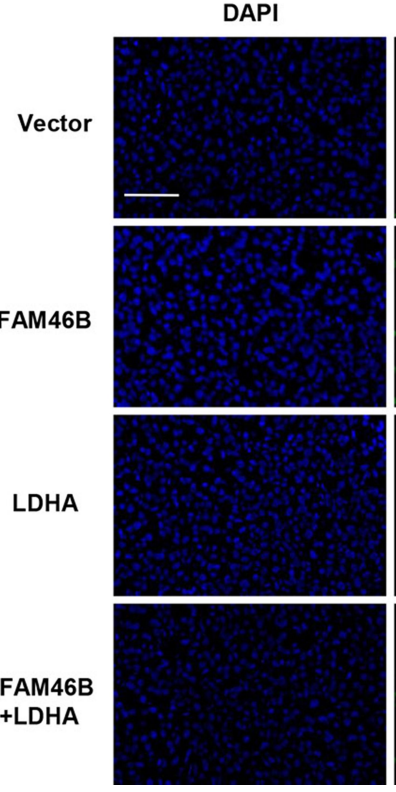

B
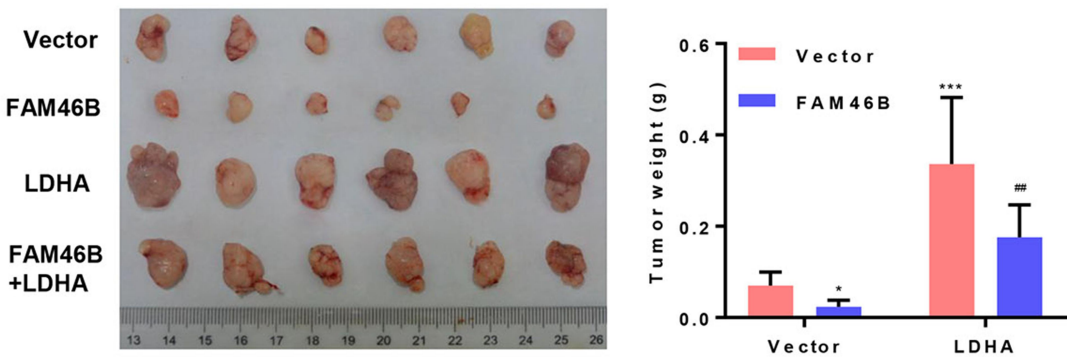

D

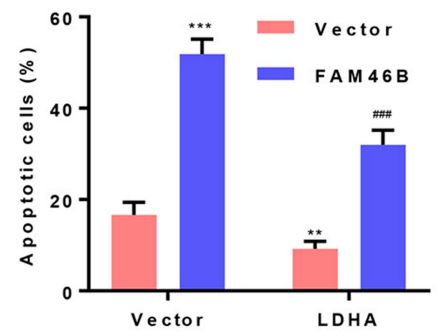

E

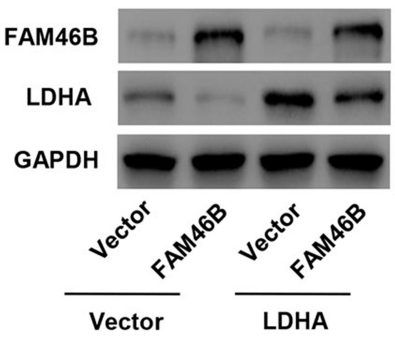

Figure 5 FAM46B inhibits tumor growth in vivo through inhibition of LDHA. LNCaP cells stably transduced with the indicated plasmids were subcutaneously injected into nude mice ( $n=6$ per group). (A) Tumor volume was evaluated every 3 days for 33 days. (B) At day 33, mice were sacrificed, and tumors were photographed and weighed. (C and D) Xenograft tumors with TUNEL staining. Scale bar: $50 \mu \mathrm{m}$. (E) Protein expression of FAM46B and LDHA was measured by Western blot. $* P<0.05$, $* * P<0.01$, $* * * P<0.001$ compared with vector group. ${ }^{\#} P<0.01,{ }^{m} P<0.001$ compared with FAM46B group. 
A

LDHA mRNA level $(P<0.001)$

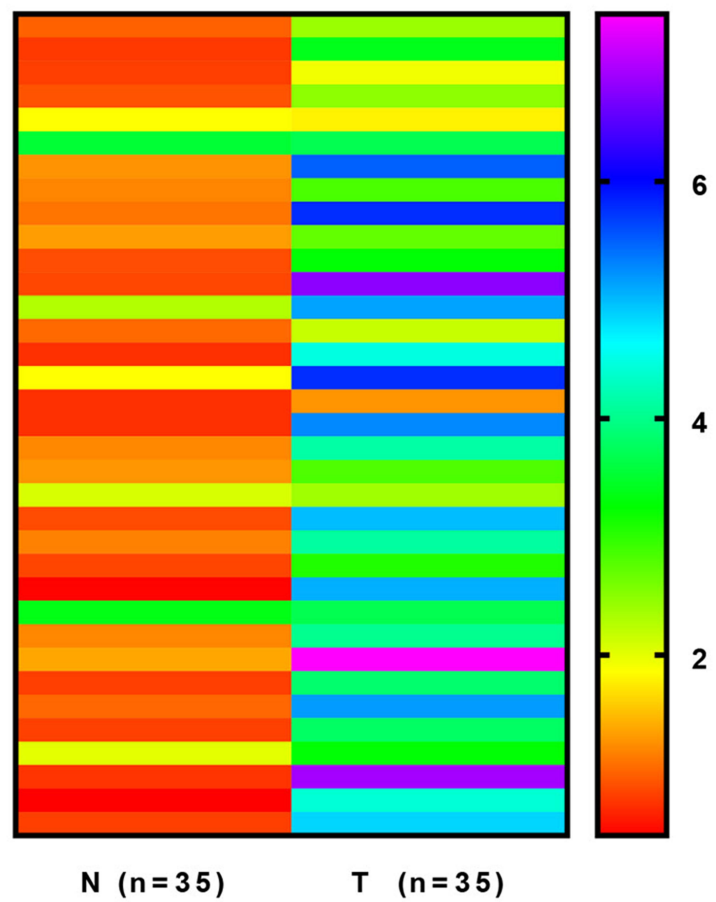

B

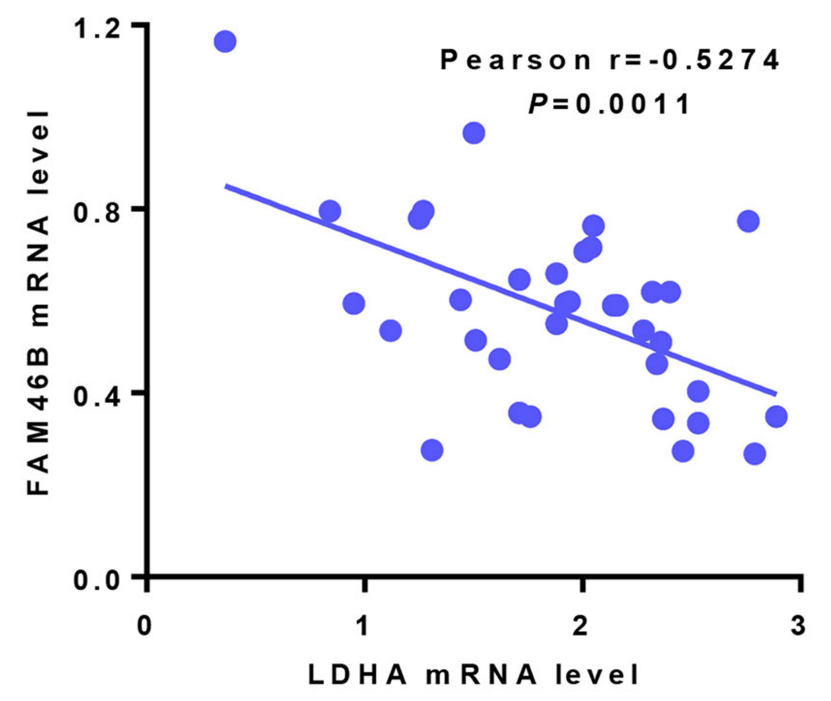

Figure 6 Correlation analyses in prostate cancer tissues. (A) LDHA mRNA expression in 35 prostate cancer (T) and paired normal (N) tissues from the hospital cohort. (B) Pearson correlation scatter plot in prostate cancer tissues $(n=35)$.

primary prostate cancer samples expressing high levels of FAM46B correlated to extended overall survival in patients compared to those expressing lower levels of FAM46B. The hypothesis that FAM46B may play a role in apoptosis was confirmed through manipulation of cell lines which showed that high FAM46B expression correlated with increased apoptosis and reduced glycolysis while elevated LDHA has the opposite impact. This pattern poses two potential opportunities. First, these data highlight the utility of screening prostate cancer patients for FAM46B expression. Given the above, more aggressive treatment may be beneficial for those with low FAM46B expression. Second, restoration of FAM46B abundance via gene therapy or other mechanism presents a promising avenue for prostate cancer treatment.

In our study, we found FAM46B expression correlates negatively with tumor size and TNM stage and positively correlates with favorable prognosis in patients with prostate cancer. Mechanistically, FAM46B clearly plays a role in regulating apoptosis in prostate cancer. Likewise, we have demonstrated here that FAM46B also mediates glycolysis. While FAM46B potentially has multiple avenues for cell killing, these data suggest a likely scenario that involves a mechanism of inducing apoptosis through "starving" cancer cells by downregulating glycolysis. Our data show that this mechanism is mediated via LDHA downregulation. Together, these results indicate that FAM46Bmediated LDHA downregulation can inhibit tumor growth.

Much like FAM46B, LDHA holds a central role in regulating apoptosis in prostate cancer. Here, we show that increased LDHA expression reduced apoptosis and counteracted the pro-apoptotic effect of FAM46B. As expected, overexpression of LDHA increased glucose uptake and increased cellular lactate levels, functionally enhancing the Warburg Effect. Similarly, LDHA expression and LDH level were increased in prostate cancer samples and were prognostic indicators for prostate cancer patients with metastasis. ${ }^{28-30}$ Furthermore, downregulation of LDHA also suppresses prostate cancer cell proliferation, migration, and invasion, increases cell apoptosis and reprograms glucose metabolism. ${ }^{28}$

LDHA protein abundance and total LDH activity are suppressed by the presence of FAM46B. We also show that MYC is also opposed by FAM46B; MYC has been shown to regulate LDHA in lymphoid cells ${ }^{26}$ and it stands to reason that a similar mechanism may be at play here. Other potential regulators of LDHA include $\beta$-catenin, ${ }^{31}$ which has been proposed to regulate $\mathrm{MYC}^{32}$ and cyclin $\mathrm{G} 2,{ }^{22}$ among others. 
As we have shown MYC to be regulated by FAM46B, and our previous study reported that FAM46B inhibits cell proliferation and cell cycle progression in prostate cancer through ubiquitination of $\beta$-catenin, ${ }^{7}$ it is probable that FAM46B may regulate LDHA expression via the $\beta$-catenin /MYC signaling in prostate cancer. LDHA has been known to be involved throughout oncogenesis ${ }^{33}$ particularly due to the increased energy needs of cancer cells. ${ }^{15}$ Conversely, inhibition of LDHA in cancer causes mitochondrial dysfunction in breast cancer. ${ }^{34}$ It was therefore not surprising to find increased expression of LDHA in the prostate cancer samples examined here and loss of LDHA correlating with the induction of apoptosis is compatible with glycolysis-dependence. However, as MYC regulates multiple gene expression profiles, we cannot conclusively determine this phenotype is LDHA-dependent. Additionally, LDHA expression is regulated by other transcription factors $(\mathrm{eg} \mathrm{HIF} 1 \alpha)^{35}$ in prostate cancer which further confounds connecting $\mathrm{MYC}$ and LDHA. The interwoven regulatory pathways emanating from LDHA will need further analysis to fully describe the role of LDHA in mediating the Warburg effect.

\section{Conclusion}

The data here present a framework for the understanding of the FAM46B/LDHA axis in prostate cancer. Further research will need to be performed to elucidate the specific mechanisms at play regarding the roles of FAM46B and LDHA in prostate cancer. The specific role of MYC and its relationship with $\beta$-catenin should be further defined. The mechanism by which LDHA inhibits cleaved caspase- 3 has yet to be defined but could serve as a keystone therapeutic target as this is where a connection between energy metabolism and apoptosis resides. Possibly most important is defining the cause of FAM46B suppression in aggressive prostate cancer as these data may highlight an important genetic or environmental risk factor.

\section{Funding}

This study was supported by the Scientific Research Fund of Shanghai Sixth People's Hospital East campus (DY2018008).

\section{Disclosure}

The authors report no conflicts of interest in this work.

\section{References}

1. Bray F, Ferlay J, Soerjomataram I, Siegel RL, Torre LA, Jemal A. Global cancer statistics 2018: GLOBOCAN estimates of incidence and mortality worldwide for 36 cancers in 185 countries. CA Cancer $J$ Clin. 2018;68(6):394-424. doi:10.3322/caac.21492

2. Negoita S, Feuer EJ, Mariotto A, Cronin KA, Petkov VI, Hussey SK. Annual report to the nation on the status of cancer, part II: recent changes in prostate cancer trends and disease characteristics. Cancer. 2018;124(13):2801-2814. doi:10.1002/cncr.31549

3. Jemal A, Bray F, Center MM, Ferlay J, Ward E, Forman D. Global cancer statistics, 2012. CA Cancer J Clin. 2011;61(2):69-90. doi: $10.3322 /$ caac. 20107

4. Long J, Zhang B, Signorello LB, et al. Evaluating genome-wide association study-identified breast cancer risk variants in African-American women. PLoS One. 2013;8(4):e58350. doi:10. 1371/journal.pone. 0058350

5. Etokebe GE, Zienolddiny S, Kupanovac Z, et al. Association of the FAM46A gene VNTRs and BAG6 rs3117582 SNP with non small cell lung cancer (NSCLC) in Croatian and Norwegian populations. PLoS One. 2015;10(4):e0122651. doi:10.1371/journal.pone.0122651

6. Özdemir TR, Şimşir A, Onay H, Cüreklibatır İ, Özkınay F, Akın H. Whole-genome gene expression analysis in urine samples of patients with prostate cancer and benign prostate hyperplasia. Urol Oncol. 2017;35(10):607.e615-607.e624. doi:10.1016/j.urolonc.2017.05.020

7. Liang T, Ye X, Liu Y, et al. FAM46B inhibits cell proliferation and cell cycle progression in prostate cancer through ubiquitination of $\beta$-catenin. Exp Mol Med. 2018;50(12):1-12. doi:10.1038/s12276-018-0184-0

8. Zhu YX, Shi CX, Bruins LA, et al. Loss of FAM46C promotes cell survival in myeloma. Cancer Res. 2017;77(16):4317-4327. doi:10.1158/0008-5472.CAN-16-3011

9. Mroczek S, Chlebowska J, Kuliński TM, et al. The non-canonical poly(A) polymerase FAM46C acts as an onco-suppressor in multiple myeloma. Nat Commun. 2017;8(1):619. doi:10.1038/s41467-01700578-5

10. Zhang QY, Yue XQ, Jiang YP, Han T, Xin HL. FAM46C is critical for the anti-proliferation and pro-apoptotic effects of norcantharidin in hepatocellular carcinoma cells. Sci Rep. 2017;7(1):396. doi:10.1038/s41598-017-00313-6

11. Wan XY, Zhai XF, Jiang YP, Han T, Zhang QY, Xin HL. Antimetastatic effects of norcantharidin on hepatocellular carcinoma cells by up-regulating FAM46C expression. Am J Transl Res. 2017;9 (1):155-166.

12. Bettoni F, Filho FC, Grosso DM, et al. Identification of FAM46D as a novel cancer/testis antigen using EST data and serological analysis. Genomics. 2009;94(3):153-160. doi:10.1016/j.ygeno.2009.06.001

13. Warburg O, Wind F, Negelein E. The metabolism of tumors in the body. J Gen Physiol. 1927;8(6):519-530. doi:10.1085/jgp.8.6.519

14. He TL, Zhang YJ, Jiang H, Li XH, Zhu H, Zheng KL. The c-MycLDHA axis positively regulates aerobic glycolysis and promotes tumor progression in pancreatic cancer. Med Oncol. 2015;32(7):187. doi:10.1007/s12032-015-0633-8

15. Schwartsburd P. Cancer-induced reprogramming of host glucose metabolism: "vicious cycle" supporting cancer progression. Front Oncol. 2019;9:218. doi:10.3389/fonc.2019.00218

16. Huang X, Li X, Xie X, et al. High expressions of LDHA and AMPK as prognostic biomarkers for breast cancer. Breast. 2016;30:39-46. doi:10.1016/j.breast.2016.08.014

17. Li L, Kang L, Zhao W, et al. miR-30a-5p suppresses breast tumor growth and metastasis through inhibition of LDHA-mediated Warburg effect. Cancer Lett. 2017;400:89-98. doi:10.1016/j. canlet.2017.04.034

18. Hu S, Jiang Q, Luo D, et al. miR-200b is a key regulator of tumor progression and metabolism targeting lactate dehydrogenase $\mathrm{A}$ in human malignant glioma. Oncotarget. 2016;7(30):48423-48431. doi:10.18632/oncotarget.10301 
19. Zhu W, Ma L, Qian J, et al. The molecular mechanism and clinical significance of LDHA in HER2-mediated progression of gastric cancer. Am J Transl Res. 2018;10(7):2055-2067.

20. Urbańska K, Orzechowski A. Unappreciated role of LDHA and LDHB to control apoptosis and autophagy in tumor cells. Int $\mathrm{J} \mathrm{Mol}$ Sci. 2019;20(9):2085. doi:10.3390/ijms20092085

21. Liu J, Chen G, Liu Z, et al. Aberrant FGFR tyrosine kinase signaling enhances the Warburg effect by reprogramming LDH isoform expression and activity in prostate cancer. Cancer Res. 2018;78 (16):4459-4470. doi:10.1158/0008-5472.CAN-17-3226

22. Li S, Gao J, Zhuang X, et al. Cyclin G2 inhibits the Warburg effect and tumour progression by suppressing LDHA phosphorylation in glioma. Int J Biol Sci. 2019;15(3):544-555. doi:10.7150/ijbs.30297

23. Shi M, Cui J, Du J, et al. A novel KLF4/LDHA signaling pathway regulates aerobic glycolysis in and progression of pancreatic cancer. Clin Cancer Res. 2014;20(16):4370-4380. doi:10.1158/1078-0432. CCR-14-0186

24. Cui J, Shi M, Xie D, et al. FOXM1 promotes the warburg effect and pancreatic cancer progression via transactivation of LDHA expression. Clin Cancer Res. 2014;20(10):2595-2606. doi:10.1158/ 1078-0432.CCR-13-2407

25. Tao R, Murad N, Xu Z, Zhang P. MYC drives group 3 medulloblastoma through transformation of Sox $2(+)$ astrocyte progenitor cells. Cancer Res. 2019;79(8):1967-1980. doi:10.1158/0008-5472.CAN-18-1787

26. Shim H, Dolde C, Lewis BC, et al. c-Myc transactivation of LDH-A: implications for tumor metabolism and growth. Proc Natl Acad Sci U S A. 1997;94(13):6658-6663. doi:10.1073/pnas.94.13.6658

27. Das L, Vinayak M. Curcumin modulates glycolytic metabolism and inflammatory cytokines via Nrf 2 in dalton's lymphoma ascites cells in vivo. Anticancer Agents Med Chem. 2018;18(12):1779-1791. doi:10.2174/1871520618666180604093802
28. Xian ZY, Liu JM, Chen QK, et al. Inhibition of LDHA suppresses tumor progression in prostate cancer. Tumour Biol. 2015;36 (10):8093-8100. doi:10.1007/s13277-015-3540-X

29. Naruse K, Yamada Y, Aoki S, et al. Lactate dehydrogenase is a prognostic indicator for prostate cancer patients with bone metastasis. Hinyokika kiyo. Acta urologica Japonica. 2007;53(5):287-292.

30. Findakly D, Wang J. Intricate interplay of entwined metabolic and inflammatory life-threatening processes in tumor lysis syndrome complicating prostate cancer: a systematic review with a single institution experience. Cureus. 2020;12(3):e7395.

31. Sherwood V, Chaurasiya SK, Ekström EJ, et al. WNT5A-mediated $\beta$ catenin-independent signalling is a novel regulator of cancer cell metabolism. Carcinogenesis. 2014;35(4):784-794. doi:10.1093/carcin/bgt390

32. Fang Y, Shen ZY, Zhan YZ, Feng XC, Chen KL, Li YS. CD36 inhibits $\beta$-catenin/c-myc-mediated glycolysis through ubiquitination of GPC4 to repress colorectal tumorigenesis. Nat Commun. 2019;10 (1):3981. doi:10.1038/s41467-019-11662-3

33. Mohammad GH, Olde Damink SW, Malago M, Dhar DK, Pereira SP, Muders M. Pyruvate kinase M2 and lactate dehydrogenase A are overexpressed in pancreatic cancer and correlate with poor outcome. PLoS One. 2016;11(3):e0151635. doi:10.1371/journal.pone.0151635

34. Wang ZY, Loo TY, Shen JG, et al. LDH-A silencing suppresses breast cancer tumorigenicity through induction of oxidative stress mediated mitochondrial pathway apoptosis. Breast Cancer Res Treat. 2012;131(3):791-800. doi:10.1007/s10549-011-1466-6

35. Tsui KH, Chung LC, Wang SW, Feng TH, Chang PL, Juang HH. Hypoxia upregulates the gene expression of mitochondrial aconitase in prostate carcinoma cells. J Mol Endocrinol. 2013;51(1):131-141. doi:10.1530/JME-13-0090

\section{Publish your work in this journal}

OncoTargets and Therapy is an international, peer-reviewed, open access journal focusing on the pathological basis of all cancers, potential targets for therapy and treatment protocols employed to improve the management of cancer patients. The journal also focuses on the impact of management programs and new therapeutic agents and protocols on patient perspectives such as quality of life, adherence and satisfaction. The manuscript management system is completely online and includes a very quick and fair peer-review system, which is all easy to use. Visit http://www.dovepress.com/ testimonials.php to read real quotes from published authors 